\title{
BMJ Open Creating accountable hospital service areas in China: a case analysis of health expenditure in the metropolis of Chengdu
}

\author{
Peiya Cao, ${ }^{1,2}$ Xiaoshuang Zhao, ${ }^{3}$ Yili Yang, ${ }^{2}$ Jay Pan (1) ${ }^{1,2}$
}

To cite: Cao P, Zhao X, Yang $Y$, et al. Creating accountable hospital service areas in China: a case analysis of health expenditure in the metropolis of Chengdu. BMJ Open 2022;12:e051538. doi:10.1136/ bmjopen-2021-051538

- Prepublication history and additional supplemental material for this paper are available online. To view these files, please visit the journal online (http://dx.doi.org/10.1136/ bmjopen-2021-051538).

Received 22 March 2021 Accepted 10 January 2022

\section{Check for updates}

(C) Author(s) (or their employer(s)) 2022. Re-use permitted under CC BY-NC. No commercial re-use. See rights and permissions. Published by BMJ.

${ }^{1}$ West China School of Public Health and West China Fourth Hospital, Sichuan University, Chengdu, Sichuan, China ${ }^{2}$ HEOA Group, Institute for Healthy Cities and West China Center for Rural Health Development, Sichuan University, Chengdu, Sichuan, China

${ }^{3}$ Shenzhen Longgang Chronic Disease Hospital, Shenzhen, Guangdong, China

Correspondence to

Professor Jay Pan;

panjie.jay@scu.edu.cn

\section{ABSTRACT}

Objectives To delineate hospital service areas (HSAs) using the Dartmouth approach in China and identify the hypothesised demand-side, supply-side and regionspecific factors of health expenditure within HSAs.

Design Population-based descriptive study.

Setting We selected the metropolis of Chengdu, one of the three most populous cities in China as a case for the analysis, where approximately 16.33 million residents living.

Participants Individual-level in-patient discharge records ( $n=904298$ ) during the fourth quarter of 2018 (from 1 September to 31 December) were extracted from Sichuan Health Commission. Cases of non-residents of Chengdu were excluded from the datasets.

Methods We conducted three sets of analyses: (1) apply Dartmouth approach to delineate HSAs; (2) use Geographic Information System (GIS)-based method to demonstrate health expenditure variations across delineated HSAs and (3) employ a three-level multilevel linear model to examine the association between health expenditure and demandside, supply-side and region-specific factors.

Results A total of 113 HSAs with a median population of 60472 (ranging from 7022 to 827 750) was delineated. Total in-patient expenditure per admission varied more than threefold across HSAs after adjusting for age and gender. Apart from a list of demand-side factors, an increased number of physicians, healthcare facilities at higher levels and for-profit healthcare facilities were significantly associated with increased total in-patient expenditures. At the HSA level, the proportion of private healthcare facilities located in a single HSA was associated with increased total in-patient expenditure generated by that HSA, while the increased number of healthcare facilities in a HSA was negatively associated with the total in-patient expenditures.

Conclusion HSAs were delineated to help establish an accountable healthcare delivery system, which serves as local hospital markets to provide in-patient healthcare via connecting demanders with suppliers inside particular HSAs. Policy-makers should adopt HSAs to identify variations of total in-patient expenditures among different areas and the potential associated factors. Findings from the HSA-based analysis could inform the formulation of relevant health policies and the optimisation of healthcare resource allocations.
Strengths and limitations of this study

To delineate hospital service areas (HSAs) using the Dartmouth approach in China.

- We applied Geographic Information System (GIS)based methods to demonstrate significant variations in healthcare expenditure across HSAs.

- Significant inconsistencies embedded in healthcare resource allocations from demanders' and suppliers' perspectives were identified.

- Our estimated patterns for using in-patient services based on our findings might probably lack stability with possible fluctuations due to the availability of quarterly discharge data.

\section{INTRODUCTION}

The remarkable economic development in China over the past decades has induced a dramatical increase in national health expenditures, especially during the past 10 years when the nationwide demand for healthcare has been significantly stimulated by the penetration of universal health insurance coverage since the initiation of China's new phase of national healthcare reform in 2009, which drastically increased from $5.15 \%$ ( $¥ 17$ 541.9 billion) of the gross domestic product (GDP) in 2009 to $6.58 \%$ in 2019 (¥65 195.9 billion). ${ }^{1}$ Although China spends less on health as a share of its national GDP than most Organisation for Economic Co-operation and Development (OECD) countries, the growth rate of such expenditure on health as a share of the national GDP presented to be higher than that of all OECD countries over the past decades, ${ }^{2}$ which therefore has compromised the affordability of medical costs for nationwide residents in China, thus posing an immense financial burden on the national medical insurance programme as well as on the whole society. As a result, the implementation of effective strategies aimed at curtailing medical costs for 
residents has been highlighted as an essential goal to be achieved throughout the latest phase of China's healthcare reform. In such context, this study aimed to identify potential factors associated with dramatically increasing health expenditures via the creation of population-based hospital services areas (HSAs) as an innovatively developed approach based on previous studies.

As indicated by previous studies, an increased amount of health services per case of the disease has been identified as a potential stimulant for increased health expenditures, which is highly associated with a list of factors induced by the demand side ${ }^{3}$ such as the prevalence or incidence of diseases, population ageing, population growth, cultural habits, opportunity costs, income or preferences. ${ }^{45}$ However, some of these demand-side factors are typically difficult to observe and measure. The literature mainly focuses on the supply-side factors ${ }^{6}$ and regionspecific factors. ${ }^{7-9}$ In an attempt to explore the potential causes underlying the increase of health expenditures among different regions, a map of HSAs was created by previous studies in order to demonstrate how hospitals were used among various areas as well as depicting where most local hospitalisation occurred based on the consideration of different travel patterns for accessing hospital services among residents across the entire regions. ${ }^{10-12}$ As most of the patients living in a certain HSA would typically seek health services from hospitals that are in the same HSA, thus serving as coherent in terms of connections between supply and demand for hospital service and because of this, this HSA-based approach has been considered as helpful for analysing the accountability of different healthcare organisations in terms of affecting the total health expenditures across regions. Specifically, as hospitals located in the same HSA would contribute to the overall health expenditure within that particular HSA, conducting analysis on hospitals based on the HSA map would serve as an ideal approach for investigating health expenditure variations among different regions. ${ }^{13}$

Researchers have already widely adopted this HSAbased approach to demonstrate variations in health expenditures among different regions. ${ }^{14-18}$ Surprisingly, these studies found that most geographic variations in health expenditures are not driven by patient characteristics, such as the prevalence of patient illness, severity of disease, and patient preferences for seeking medical services. On the contrary, practice style differences, diagnostic and therapeutic uncertainties in clinical medicine, and more 'supply-sensitive care' are the most widely employed hypotheses to explain these variations. ${ }^{19}$ Based on such evidence, several health policies implemented in the USA aimed to reduce payments in high-expenditure areas based on the previously developed HSA map in which hospitals within the same HSA were considered accountable for the total health expenditure in that particular region.

It is noteworthy that the development of a suitable area model serves as an indispensable component to be analysed on a unit basis while examining the accountability of healthcare organisations among different regions. Administrative units such as counties, states, and nations are typically used for analysis considering both feasibility and policy issues. However, it should be noted that a list of potential factors independent of administrative boundaries might also have a significant impact on utilisation patterns among residents, such as geographical distributions of healthcare institutions, travel time needed for accessing medical services, healthcare infrastructure constructions among different regions as well as residents' preference for obtaining medical services from particular healthcare institutions. ${ }^{20}$ As a consequence, it is common to find residents seeking medical services across different administrative boundaries, ${ }^{21}$ which would add to the difficulty of assessing the pattern of health services utilisation among residents who prefer to seek medical services outside their residential areas due to the numerator-denominator mismatch issue. ${ }^{22}$ Therefore, the value of evaluating health expenditures based on the HSAs approach mainly resides in that a list of potential factors independent of administrative boundaries can be investigated via this procedure, which might significantly impact health services utilisation patterns among residents across different administrative units.

Despite the great value of adopting HSAs as an ideal approach for evaluating health expenditures among different areas, the actual employment of this innovative approach remains limited in China and other lowincome and middle-income countries (LMICs). Three leading factors could explain this as the primary reasons. First, health service systems in LMICs remain relatively underdeveloped, for which insufficient provision of health services should be addressed as the primary issue. In such a context, policymakers in those countries have been focused on increasing the number of healthcare providers instead of improving the quality of healthcare outputs, as reflected by increased health expenditures with poorly improved healthcare outcomes. ${ }^{23}$ In addition, LMICs remain relatively backward in conducting health policies research, for which a list of methods widely adopted by developed countries for both study and health-related policy-making purposes have not been well adopted yet to serve such purposes in LMICs, especially in the aspect of providing evidence-based implications for health-related policy-making and decision-making procedures via the adoption of well-established models such as the HSA-based approach. Last but not least, despite the rapid development of health systems in multiple countries with an increased focus on associating the accountability of healthcare organisations directly with healthcare outcomes, the underdevelopment of nationwide health information systems in those countries have posed a huge hindrance in obtaining reliable diagnostic and therapeutic information recorded from patients to facilitate the adoption of the HSA-based approach. ${ }^{24}$

Based on the previously developed HSAs delineation approaches, this study applied the classic Dartmouth approach to help establish an accountable healthcare 
delivery system in China. We selected the metropolis of Chengdu, one of the three most populated cities in China, as a case for the analysis with approximately 16.33 million residents. Based on the delineated HSAs, our findings are expected to identify a list of potential contributors to health expenditure variations among different regions to provide evidence-based implications for improving the nationwide healthcare system with increased affordability of medical costs for national patients. Our first contribution would be the first study to delineate HSAs in China and reference its future application. The second contribution would be to demonstrate health expenditure variations across delineated HSAs and identify the hypothesised demand-side, supply-side and regionspecific factors for health expenditures in China.

\section{MATERIAL AND METHODS}

\section{Study area}

We selected Chengdu as the study area to create accountable hospital service areas (HSAs). As the capital city of Sichuan province located in the southwest of China with an area of $14335 \mathrm{~km}^{2}$, Chengdu has 16.33 million residents with its GDP per capita reported as $¥ 94782$ (US\$14 444 ) in 2018. Its total population and GDP ranked as 2th and 47th among all the Chinese prefecture-level cities $(278) .{ }^{25}$ The medical infrastructures have been well constructed in this study area, most $(98.21 \%)$ of the residents in this study area typically choose to seek medical services within the city under a well-established government-led health information system, thus providing us with an excellent opportunity for creating an HSAs map to describe the accountabilities of various healthcare organisations across the study area.

Figure 1 shows the location, population density and distribution of medical institutions which provided in-patient services in Chengdu. Chengdu has 20 districts with 375 communities. The downtown of Chengdu is located in the city's central area with a densely clustered population distribution. Despite that the distribution of hospitals covered a wide range of locations across the city, a higher concentration of institutions was mainly found in the central area, particularly tertiary hospitals.

\section{Data sources}

We extracted hospital discharge data collected at the health administrative level during the fourth quarter of 2018 (from 1 September to 31 December) from the Sichuan Health Commission. The data sets included all hospital discharges for the entire population residing in Chengdu over the study period, which contained over 900 thousand hospitalisation discharge cases. For each patient admitted to the hospital, the patient's specific place of residence would be identified by community codes and recorded as part of discharge records. At the same time, a unique numerical identifier would be produced for recording where the admission took place. Such recording procedures

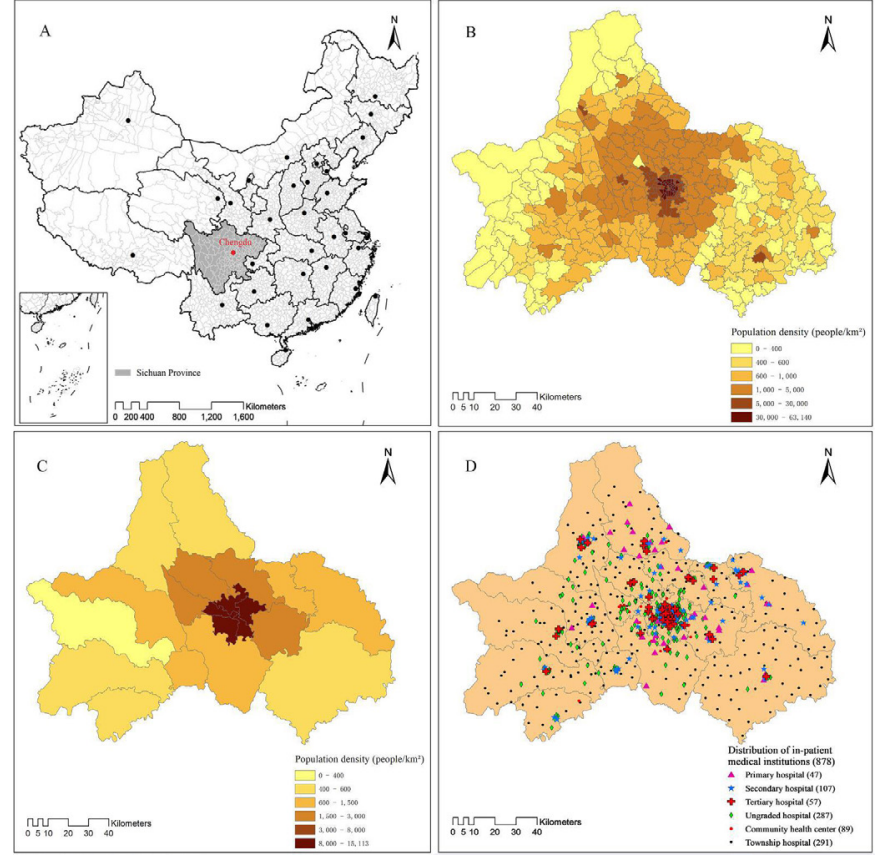

Figure 1 Location (A), population density by the community (B) and by district (C) of the metropolis of Chengdu, China; distribution of in-patient medical institutions used by patients (D) (Based on the data collected from the health and family planning Commission of Sichuan Province, communities include township, towns and street communities, the listed maps were developed under ArcGIS V.10.2 environment).

underlying the discharge records served as a reliable basis for determining the geographic patterns of medical service utilisation. Individual hospitalisation cases were considered the units for observations, while patients' discharge cases from outside Chengdu were excluded from the datasets to improve the stability of estimations for discharge patterns. After these data screening procedures, 904298 discharge cases in Chengdu were finally retained for analysis.

The hospital-level data were extracted from hospitals and primary healthcare institutions' (township hospitals, THs; and community health centres, CHCs) annual reports provided by Sichuan Health Commission. Several variables were used to describe the attributes of each healthcare facility, including a unique numerical identifier, the community where the facility was located, the number of physicians and the number of hospital beds.

\section{Patient and public involvement}

Patients or members of the public were not involved in the design and other stages of this study. Health resourcing data are available in a public access repository. Healthcare services data of patients were routinely collected by the health administration department and were aggregated at the community level, and all individuals' personal information has been desensitised. Therefore, it could not be identified from this analysis. 


\section{Definition of HSAs}

Steps for HSAs delineation

HSAs were defined through three steps in our study based on the original Dartmouth approach. First, when replicating the Dartmouth approach, we geocoded locations of facilities providing in-patient services by community codes instead of town or city code to define HSAs at a finer scale. Hospitals and primary healthcare institutions are the principal owners in charge of managing healthcare resources and major providers of healthcare services in China. ${ }^{26}$ Therefore, all hospitals and primary healthcare institutions (including THs, CHCs) which provide in-patient services in the 375 communities were identified from the discharge file and assigned to the community where they resided at the time of admission. In the second step, we first geocoded patient residences by community codes. Next, using a plurality rule, each community code was assigned to that community code containing the facilities most often used by residents. All hospital/primary health institution discharge records were analysed to assign a unique community code to each of its admitted patients. A total of $724794(80.15 \%)$ of individual residences can be accurately associated with particular community codes. While other 179504 records had to be randomly assigned to all communities due to the inaccuracy of their residential locations based on the hospital discharge data. When a community had more than one hospital, the counts were added together. The third step was to visually examine the community codes to make sure that the community codes included in the HSAs were contiguous: reassigning each disconnected community code to an adjacent HSA to ensure the geographic contiguity of all community codes in one HSA; this is because the contiguity is a geographical convention, ensures the readability and interpretability of maps. ${ }^{27}$

The propensity of patients to use local hospitals is measured by localisation index (LI), the percentage of all hospitalisations in local hospitals. ${ }^{28}$ It is a populationbased index and calculated by dividing discharges of HSA-residents within their HSA by the local discharges of HSA residents. Population size, number of in-patient care facilities, health resources, including the number of beds per 1000 population and physicians per 1000 population, were also calculated. After this step, health expenditure variations across delineated HSAs were described by the total in-patient expenditure per admission. To account for the difference in population characteristics across HSAs, we adjusted for demographic factors including age (from $\leq 14$ years of age, $15-64$ and $\geq 65$ according to the age composition of permanent residents of Chengdu in 2018 and gender.

\section{Statistical analysis}

As an attempt to identify potential contributors to the variations of total in-patient expenditure across different accountable HSAs, a three-level multilevel linear model was established to explore factors influencing total in-patient expenditure with the unit of analysis at the patient level incorporating supply and region-specific covariates at the facility and HSA level. The model is set as follows:

$$
\log \left(E_{i j k}\right)=\delta_{0 j k}+\text { Patient }_{i j k}^{\prime} \pi+\text { Facility }_{j k}^{\prime} \gamma+H S A_{k}^{\prime} \lambda+e_{0 i j k}
$$

Eq.1

$$
\begin{gathered}
\delta_{o j k}=\delta_{00 k}+\nu_{0 j k} \\
\nu_{0 j k}=\pi_{000+} \nu_{00 k} \\
\nu_{00 k} \sim N(0,) \\
\nu_{o j k} \sim N\left(0, \sigma_{v 0}^{2}\right) \\
e_{0 i j k} \sim N\left(0, \sigma_{e 0}^{2}\right)
\end{gathered}
$$

Where patients were at level 1 , facilities were at level 2 and HSAs were at level 3. $E_{i j k}$ was the explained variable, which denoted the total in-patient expenditure for individual $i$ treated in facility $j$ in HSA $k$. The total in-patient expenditures were natural log-transformed before regression analysis because they were right-skewed. The intercept $\delta_{0 j k}$ indicated the average logarithm of health expenditure between 878 healthcare facilities and 113 HSAs; $v_{0 j k}$ and $v_{0 o k}$ were the terms of facility-level and HSAlevel random effects, respectively. $e_{i j}$ was the error term. Patient ${ }_{i j k}$ denoted a vector of individual's characteristics including age, gender, health insurance programme (Urban Employment Basic Medical Insurance, Urban Residents Basic Medical Insurance, New Cooperative Medical Scheme, full self-expense and others), Occupation (thirteen categories), Charlson Comorbidity Index which was calculated through all secondary diagnoses, classification of 22 diseases (online supplemental appendix 1) which was grouped by the first three characters of 10th version of International Classification of Diseases, the urgency at the admission (critical urgent, urgent, general). Facility' ${ }_{j k}$ denoted a vector of supply-side variables, including healthcare facility level, ownership, whether for-profit and the number of physicians. HSA'k denoted a vector of HSA-specific variables, including physicians per 1000 persons, proportions of physicians in tertiary hospitals, the number of in-patient care facilities, proportions of private healthcare facilities, proportions of for-profit healthcare facilities and proportions of referrals outside of the city in HSAs. The definition of all variables can be found in online supplemental appendix 2; in total, there were 904298 observations in the model; a two-sided $\mathrm{p}$ value is used, with $\alpha$ set to 0.05 to determine statistical significance. The model analysis was performed using R V.4.0.2.

\section{RESULTS}

\section{Overall review of HSAs}

A total of 113 HSAs were finally delineated in Chengdu, as shown in figure 2. Five HSAs were defined as a geographic extension beyond district boundaries and were mainly 


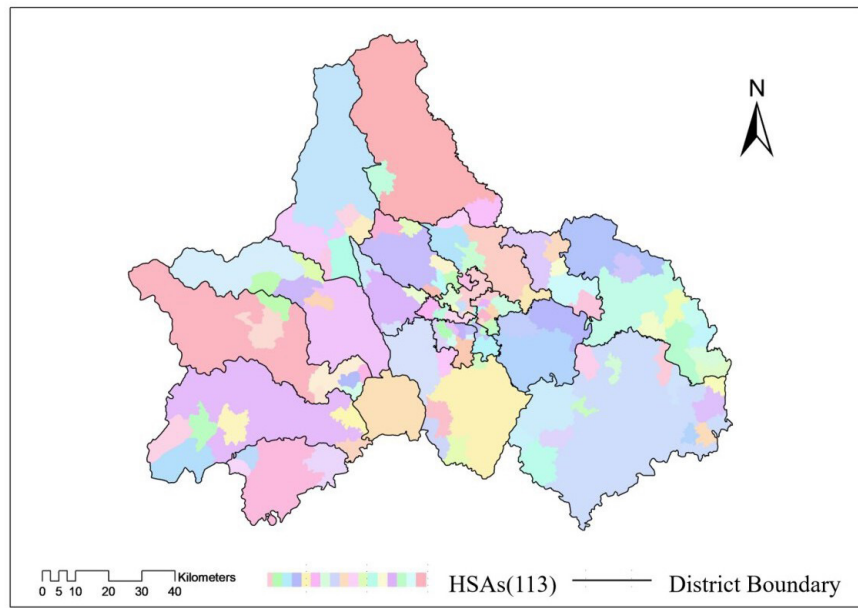

Figure 2 Accountable hospital service areas (HSAs) in the Metropolis of Chengdu, China.

displayed in the city's centre. Larger HSAs were more commonly found in the boundaries of the city.

HSAs showed significant differences in characteristics. The LI varied from a minimum value of $11.12 \%$ to $85.52 \%$ (median $51.25 \%$ ), $62.35 \%$ of residents lived in areas where the LI exceeded $50 \%$. The mean population size was 274336 (median 60,472), ranging from 7022 to 827750 . The number of in-patient care facilities per HSA ranged from 1 to 48 , with 44 HSAs having at least five in-patient facilities per HSA while 30 HSAs had only one in-patient facility per HSA. HSAs with more than 40 in-patient care facilities were mainly located around the boundaries of Chengdu except for several scattered areas coloured in dark blue distributed in the centre of the city. The number of beds per 1000 population demonstrated large variations among separate HSAs, ranging from 1.19 to 67.02, while the number of physicians per $1000 \mathrm{popu}-$ lation varied from 0.31 to 40.27 among various HSAs (figure 3).

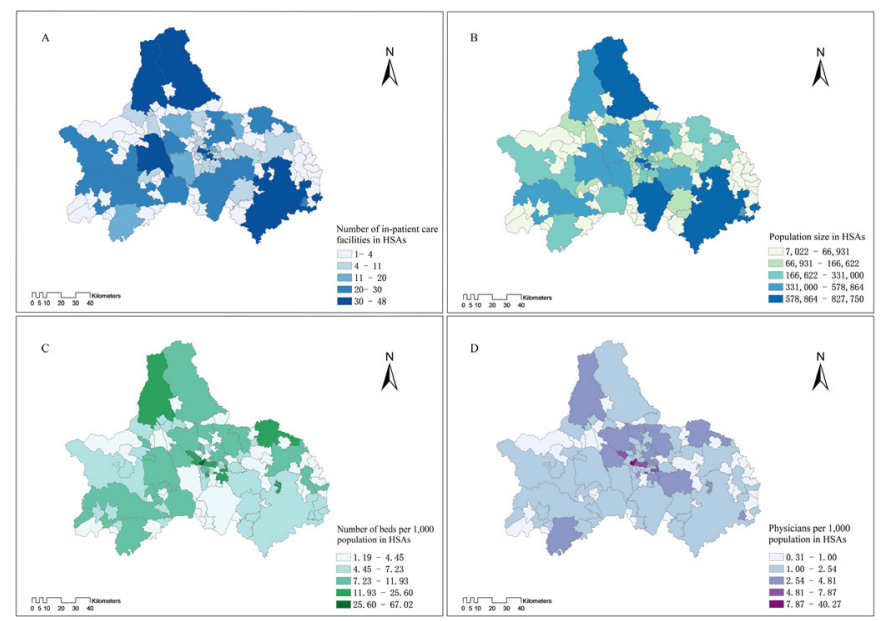

Figure 3 Number of in-patient care facilities (A) Population size (B) Number of beds (C) and physicians per 1000 population (D) in accountable hospital service areas (HSAs) in the Metropolis of Chengdu, China.

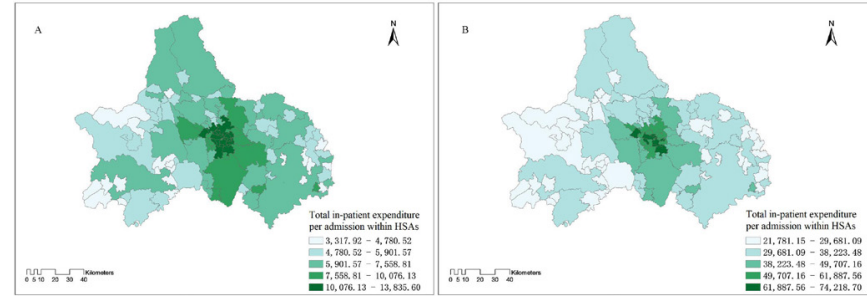

Figure 4 Total in-patient expenditure per admission among HSAs in the metropolis of Chengdu, China (A: unadjusted total in-patient expenditure per admission within HSAs; B: total in-patient expenditure per admission is age, genderadjusted expenditure). HSAs, hospital service areas.

In terms of the total in-patient expenditure per admission in 2018 among various HSAs across Chengdu, the values demonstrated more than four fold's range from $¥ 3317.92$ per admission to $¥ 13835.60$ per admission, which still showed more than threefold differences after adjusting for age and gender (figure 4). Based on the outcomes, high expenditure HSAs were clustered in the east-central part of the city.

\section{What drives health expenditure to vary across HSAs?}

Table 1 shows the results of the multilevel linear regression model, with total expenditures on in-patient services as the dependent variable. Model without covariates was the null model with no predictors that showed variations of total health expenditure among different healthcare facilities $\left(\sigma_{v 0}^{2}=0.478\right)$ and HSAs $\left(\sigma_{\mu 0}^{2}=0.023\right)$. When adjusted for patient characteristics, facility covariates, and additional HSA-specific characteristics, the model explained $53 \%$ of the variance in the total expenditure. We found that each year's increase in a single patient's age was associated with an approximately $1 \%$ increase in the total hospitalisation expenditure. Females' total in-patient expenditure was found to be about $2.8 \%$ less than males'. Regarding health insurance coverage status, patients under the coverage of URBMI, NCMS programmes, or entirely self-paid posed negative impacts on in-patient expenditures. An increase in Charlson Comorbidity Index was associated with an approximately $7.8 \%$ increase in patients' total expenditure. Moreover, the total expenditure of patients with urgent or general conditions when admitted into healthcare facilities was $24.6 \%$ and $31.4 \%$ less than patients with critically urgent conditions on admission into healthcare facilities, respectively.

From the supply side, we found that the level of healthcare facilities, whether for-profit and the number of physicians, were significantly associated with the total expenditure. The total expenditures of patients hospitalised in secondary or tertiary hospitals were $5.7 \%$ and $46.6 \%$ more than those admitted to un-graded hospitals, respectively. The total expenditures of patients hospitalised in for-profit healthcare facilities were $30.8 \%$ more than not-for-profit counterparts. In addition, every 100 increase in physicians was associated with a $10 \%$ increase in the total expenditure. 
Table 1 Factors associated with total expenditures for in-patients in the multilevel linear model

\begin{tabular}{|c|c|c|c|}
\hline \multirow[b]{2}{*}{ Model without covariates } & \multicolumn{3}{|c|}{ Log (total in-patient expenditure) } \\
\hline & Estimate $(95 \% \mathrm{Cl})$ & SE & $\operatorname{Pr}(>|t|)$ \\
\hline Intercept & 8.424 (8.395 to 8.453$)$ & 0.015 & $0.000^{\star \star *}$ \\
\hline Level 3 HSA(s)† & 0.023 & 0.151 & - \\
\hline Level 2: facility $\dagger$ & 0.478 & 0.692 & - \\
\hline \multicolumn{4}{|l|}{ Model with covariates } \\
\hline \multicolumn{4}{|l|}{ Fixed effects } \\
\hline \multicolumn{4}{|l|}{ Level 1: patient level } \\
\hline Intercept & 8.454 (8.405 to 8.503$)$ & 0.025 & $0.000^{* \star *}$ \\
\hline Age & 0.010 (0.009 to 0.010$)$ & 0.000 & $0.000^{\star * *}$ \\
\hline \multicolumn{4}{|l|}{ Gender } \\
\hline Male (reference) & 1 & - & - \\
\hline Female & $-0.028(-0.031$ to 0.024$)$ & 0.002 & $0.000^{\star \star \star}$ \\
\hline Missing & $-0.080(-0.176$ to 0.015$)$ & 0.049 & 0.100 \\
\hline \multicolumn{4}{|l|}{ Health insurance programme } \\
\hline UEBMI & 1 & - & - \\
\hline URBMI & -0.075 (-0.079 to 0.070$)$ & 0.002 & $0.000^{\star \star *}$ \\
\hline NCMS & $-0.057(-0.063$ to 0.051$)$ & 0.003 & $0.000^{* \star *}$ \\
\hline Full-self expenses & $-0.313(-0.319$ to 0.307$)$ & 0.003 & $0.000^{\star \star \star}$ \\
\hline Others & $-0.122(-0.129$ to 0.115$)$ & 0.003 & $0.000^{\star \star \star}$ \\
\hline Charlson Comorbidity Index & 0.078 (0.077 to 0.079$)$ & 0.001 & $0.000^{\star \star \star}$ \\
\hline \multicolumn{4}{|l|}{ Urgency when admission } \\
\hline Critical urgent & 1 & & \\
\hline Urgent & $-0.246(-0.253$ to 0.238$)$ & 0.004 & $0.000^{\star \star \star}$ \\
\hline General & -0.314 (-0.321 to 0.307$)$ & 0.004 & $0.000^{\star \star \star}$ \\
\hline Other control variable & Yes & - & - \\
\hline \multicolumn{4}{|l|}{ Level 2: facility level } \\
\hline \multicolumn{4}{|l|}{ Level of healthcare facility } \\
\hline Ungraded hospital & 1 & & \\
\hline Primary hospital & $-0.699(-0.748$ to 0.651$)$ & 0.025 & $0.000^{* \star *}$ \\
\hline Secondary hospital & 0.057 (0.030 to 0.085$)$ & 0.014 & $0.000^{\star \star \star}$ \\
\hline Tertiary hospital & 0.466 (0.432 to 0.499$)$ & 0.017 & $0.000^{\star \star \star}$ \\
\hline $\mathrm{TH} / \mathrm{CHC}$ & -1.007 (-1.044 to 0.970$)$ & 0.019 & $0.000^{\star \star *}$ \\
\hline \multicolumn{4}{|l|}{ Healthcare facility ownership } \\
\hline Public healthcare facility & 1 & & \\
\hline Private healthcare facility & $-0.006(-0.037$ to 0.025$)$ & 0.016 & 0.702 \\
\hline \multicolumn{4}{|l|}{ Whether for-profit } \\
\hline No & 1 & & \\
\hline Yes & 0.308 (0.278 to 0.339$)$ & 0.015 & $0.000^{\star \star *}$ \\
\hline Number of physicians & 0.001 (0.000 to 0.001$)$ & 0.001 & $0.000^{\star \star \star}$ \\
\hline \multicolumn{4}{|l|}{ Level 3: HSA level } \\
\hline Physicians per 1000 persons & $0.000(-0.003$ to 0.003$)$ & 0.002 & 0.873 \\
\hline Proportion of physicians in tertiary hospitals & $0.000(-0.001$ to 0.001$)$ & 0.001 & 0.846 \\
\hline Number of inpatient care facilities & $-0.002(-0.003$ to 0.001$)$ & 0.001 & $0.007^{\star *}$ \\
\hline Proportions of private facilities & 0.001 (0.000 to 0.002$)$ & 0.001 & $0.014^{*}$ \\
\hline Proportions of for-profit facilities & $0.000(-0.001$ to 0.000$)$ & 0.001 & 0.342 \\
\hline
\end{tabular}




\begin{tabular}{|c|c|c|c|}
\hline \multirow[b]{2}{*}{ Model without covariates } & \multicolumn{3}{|c|}{ Log (total in-patient expenditure) } \\
\hline & Estimate $(95 \% \mathrm{Cl})$ & SE & $\operatorname{Pr}(>|t|)$ \\
\hline Proportions of referrals from outside of the city & $0.001(-0.002$ to 0.002$)$ & 0.001 & 0.133 \\
\hline \multicolumn{4}{|l|}{ Random effects $†$} \\
\hline Level 3: HSA(s) & 0.001 & 0.036 & - \\
\hline Level 2: facility & 0.322 & 0.568 & - \\
\hline
\end{tabular}

The total expenditures of in-patients are natural log-transformed in the model.

${ }^{*} \mathrm{P}<0.05,{ }^{* *} \mathrm{p}<0.01,{ }^{* * *} \mathrm{p}<0.001$.

$\dagger$ indicated as variance and SD.

$\mathrm{CHC}$, community health centre; HSAs, hospital service areas; NCMS, New Cooperative Medical Scheme; TH, township hospital; UEBMI, Urban Employment Basic Medical Insurance; URBMI, Urban Resident Basic Medical Insurance.

At the HSA level, each $1 \%$ increase in the number of healthcare facilities per HSA would result in approximately a $0.2 \%$ reduction of total in-patient expenditure. Moreover, each $1 \%$ increase in the number of private healthcare facilities per HSA was found to be associated with a $0.1 \%$ increase in patients' total in-patient expenditure per HSA.

\section{DISCUSSION}

In this study, a map of 113 hospital services areas in Chengdu, China, was produced via adopting the HSAs approach previously developed by former studies based on the hospital discharge data. The HSAs depicted in our study demonstrated relatively high values of the LI, which indicated clustered distributions of in-patient services among different areas. The LI was found to be higher at the HSAs level (min: 11.12\%; max: $85.52 \%$; median: $51.25 \%$ ) than at the community level (min: $8.09 \%$, max: $84.47 \%$, median: $20.80 \%$ ). Specifically, LI is a potent indicator reflective of the validity in the HSA approach, for which higher values are desired for indicating the consistency of patients' residing areas with the locations of health services utilisation. Ideally, all in-patient care for residents is supposed to be provided within residential areas, while inevitably, there are patients seeking healthcare outside their regions. Therefore, the HSAs approach employed in our study was expected to produce a promising model capable of demonstrating residents' natural patterns in utilising hospital services and providing high LIs for possibility analysis. Based on our findings, a total of only five HSAs extended far beyond the administrative districts, which could be attributed to the abundant distributions of hospitalisation resources among various administrative districts that had enabled residents' utilisation of hospital services within their residing areas.

Another critical finding was identifying significant inconsistency embedded in healthcare resource allocations from demanders' and suppliers' perspectives. The development of the HSA map has enabled the evaluation of health resource allocations across the study area based on establishing an accountable healthcare delivery system, which demonstrated significant variations across different hospital services areas in terms of both the number of beds and physicians. Specifically, $57.52 \%$ of the HSAs in Chengdu had fewer beds (per 1000 population) than the nationwide average (6.03 per 1000 population). Besides, $80.53 \%$ of the HSAs in Chengdu had fewer physicians (per 1000 population) than that of the nationwide average (2.59 per 1000 population). The condition was even worse compared with the distribution of bed supply across the study area, as $32.74 \%$ of the HSAs were found to be equipped with highly inadequate healthcare resources, with each of those HSAs having less than one physician available for hospital service provision. Under such adverse circumstances, it would be reasonable to suggest that the allocation of healthcare resources should be optimised towards underserviced areas via the implementation of a series of strategies, such as adopting financial incentives as a tactic for attracting healthcare professionals to work in those underserviced regions. As a practical scenario of how HSAs could be adopted to facilitate health-related policy-making procedures, the HSAs approach can be used as an evidence-based guide to assist in health-related policy planning procedures. In terms of investigating the geographical variations embedded in healthcare resource allocations among different regions, which would primarily affect residents' access to hospital services, the utilisation of in-patient healthcare services, as well as related medical costs and health outcome disparities induced by such variations, the adoption of the HSAs approach is expected to provide meaningful units to be analysed for conducting studies in this field, ${ }^{11}{ }^{12}{ }^{26}$ use of in-patient care services, ${ }^{29}$ costs of care ${ }^{30}$ and the impact of these variations on health outcomes. ${ }^{31-33}$

Our study suggested significant variations of health expenditures across different areas based on the accountable healthcare delivery system established over the study area via the HSAs approach. In terms of what has induced such variations among various HSAs, notable findings were identified via the in-depth investigations into supplyside and region-specific factors. Specifically, while no significant statistical association was identified between 
physicians per 1000 people and the total in-patient expenditure in HSAs, a positive relationship was found between the increased number of physicians and the total in-patient expenditure. This finding is consistent with previous research. ${ }^{34-36}$ It is not difficult to understand that the service capacity of healthcare facilities reflected by the number of physicians would primarily affect patients' preferences for seeking medical services from particular medical institutions. Such a relationship is typically reflected as supply-sensitive care. ${ }^{37}$ This phenomenon indicates that more medical services tend to be delivered by healthcare facilities with better medical serviced capacities, thus generating more health expenditures. As a result, it was not unexpected to find that the number of physicians in facilities served as a positive contributor to the total health expenditure, which was positively associated with facilities' capacity regardless of residents' actual demand for healthcare. Based on such findings, it is highly suggested that policymakers should adopt cost-control strategies to curtail unnecessary costs induced by medical services with relatively more significant demand for physicians. Another finding is that total expenditures spent in for-profit facilities were higher than those at not-for-profit facilities. This finding may result from different pricing policies implemented for the medical services provided by for-profit and not-for-profit medical facilities in China. Specifically, while market-regulated pricing has been implemented for the former group of healthcare facilities to allow the set up of self-determined prices, governmentguided pricing has been applied for the latter group. ${ }^{38} \mathrm{By}$ implementing these different pricing policies, for-profit facilities typically have stronger profit-seeking purposes than not-for-profit ones, thereby setting up higher prices than their counterparts. Consequently, unreasonably high medical prices might be set up for medical service items associated with the adoption of high-tech facilities or tailored for specific needs of patients, which serves as a tactic to attract patients in need of sophisticated medical interventions, thus ultimately increasing health expenditures of patients.

Another interesting finding is that total in-patient expenditure was negatively associated with the number of in-patient care facilities distributed in a particular HSA, which proved to be consistent with the competitive mechanism as indicated by previous studies that intensified competition in the market would result in reduced net costs. ${ }^{39} 40$ In addition, our research found that an increased proportion of private hospitals in the hospital market was found to be positively associated with in-patient expenditure, which proved to be inconsistent with findings from a US study that an increased market share of private hospitals would reduce the medical costs for patients with acute myocardial infarction. ${ }^{41}$ It is noteworthy that a study from Norway reported that private hospitals tended to provide medical services with relatively higher profitability than public hospitals, such as coronary angiogenesis. ${ }^{42}$ Therefore, a possible explanation for our findings is that the drastic expansion of private hospitals in China over the past decade could be attributed to the latest phase of nationwide healthcare reform initiated in 2009, which aimed to stimulate the increase of medical service suppliers via encouraging the operation of healthcare institutions with the assistance of social capital resources. In an attempt to achieve this goal, encouraging the expansion and development of private hospitals has been addressed as an essential strategy throughout the national healthcare reform in order to improve the quality and efficiency of healthcare delivery from a holistic perspective. However, a series of policies aimed at facilitating private hospitals' expansion has long been controversial as private hospitals tend to provide therapy procedures for particular disease groups with relatively high-profit margins. ${ }^{43}$

Several limitations should be noted in this study. First, due to data availability, in this study, we were only able to retrieve quarterly discharge data for analysis instead of obtaining whole-year data or data collected over a certain period within several years. As a result, the estimated patterns for using in-patient services based on our findings might lack stability with possible fluctuations. Second, approximately $20 \%$ of residential locations engaged in this study failed to be associated with accurate community codes due to inadequate data quality. Therefore, this group of residents had to be randomly assigned to certain communities as their assumed residential areas, which has compromised the accuracy and reliability of the HSAs map developed in this study. Third, a correlation relationship instead of causality was established, which might result in reversed causality embedded in the total in-patient expenditure under the impacts of relevant variables. Nevertheless, this study's correlation analysis demonstrated great value by revealing factors associated with total in-patient expenditures induced from the supply and region-specific sides. Last, despite that a list of evidence was provided to depict the variation of health expenditures across HSAs, we failed to provide potent evidence on whether such variations were unwarranted, nor did we make in-depth investigations into the appropriateness of medical services delivered by hospitals.

\section{CONCLUSION}

In this study, the HSAs approach previously developed by Dartmouth was innovatively applied for helping establish an accountable medical service delivery system that covered the entire study area as a densely populated metropolis located in the largest developing country in the world. The HSAs identified served as local hospital markets for providing in-patient healthcare among different areas, which connected demanders and suppliers in particular HSAs and could be adopted for evaluating the distribution of healthcare resources from multiple aspects such as total health expenditures, medical service provision capacity, the quality of healthcare delivered as well as the variations of medical service utilisation patterns among different areas. Compared 
with previously defined administrative units in the study area, the HSAs served as finer-scaled units to be adopted throughout policy-making procedures to identify the variation of total health expenditures among different regions and the potential associated factors. Findings from the HSA-based analysis could inform the formulation of relevant health policies and the optimisation of healthcare resource allocations.

Acknowledgements The authors gratefully acknowledge David C. Goodman from The Dartmouth Institute for Health Policy \& Clinical Practice for his insightful comments to our application of HSA-based approach and we also thank Yufan Deng and Xiuli Wang for their valuable suggestions. The authors are responsible for all remaining errors.

Contributors Conceptualisation: JP; data analysis: $\mathrm{PC}$ and $\mathrm{XZ}$; funding acquisition and supervision: JP; writing-original draft: PC; writing-review and editing: PC and JP; writing-language checking: YY; guarantor: PC and JP.

Funding This study was funded by the National Natural Science Foundation of China (Grant No. 71874116 and 72074163), Ministry of Education of China (Grant No. 18YJA790062), Chengdu Federation of Social Science Association (Grant No. ZZ05), Sichuan University (Grant No. 2018hhf-27 and SKSYL201811) and China Medical Board (Grant No. 17-276).

Map disclaimer The inclusion of any map (including the depiction of any boundaries therein), or of any geographic or locational reference, does not imply the expression of any opinion whatsoever on the part of BMJ concerning the legal status of any country, territory, jurisdiction or area or of its authorities. Any such expression remains solely that of the relevant source and is not endorsed by BMJ. Maps are provided without any warranty of any kind, either express or implied.

Competing interests None declared.

Patient consent for publication Not applicable.

Ethics approval This study does not involve human participants.

Provenance and peer review Not commissioned; externally peer reviewed.

Data availability statement Data may be obtained from a third party and are not publicly available. The facility data of Chengdu that support the findings of this study are available from the Health Bureau of Sichuan Province and the Health Bureau of Chengdu, but restrictions apply to the availability of these data, which were used under license for the current study, and so are not publicly available. Data are however available from the corresponding author on reasonable request and with permission of the local Health Bureaus.

Supplemental material This content has been supplied by the author(s). It has not been vetted by BMJ Publishing Group Limited (BMJ) and may not have been peer-reviewed. Any opinions or recommendations discussed are solely those of the author(s) and are not endorsed by BMJ. BMJ disclaims all liability and responsibility arising from any reliance placed on the content. Where the content includes any translated material, BMJ does not warrant the accuracy and reliability of the translations (including but not limited to local regulations, clinical guidelines, terminology, drug names and drug dosages), and is not responsible for any error and/or omissions arising from translation and adaptation or otherwise.

Open access This is an open access article distributed in accordance with the Creative Commons Attribution Non Commercial (CC BY-NC 4.0) license, which permits others to distribute, remix, adapt, build upon this work non-commercially, and license their derivative works on different terms, provided the original work is properly cited, appropriate credit is given, any changes made indicated, and the use is non-commercial. See: http://creativecommons.org/licenses/by-nc/4.0/.

ORCID iD

Jay Pan http://orcid.org/0000-0001-9501-1535

\section{REFERENCES}

1 National Health Commission of People's Republic of China. Statistical Bulletin on the development of health care in China, 2019. Available: http://wwwnhcgovcn/guihuaxxs/s10748/202006/ ebfe31f24cc145b198dd730603ec4442shtml [Accessed October 10 2020].
2 OECD. Health at a glance 2019: OECD indicators. Paris: OECD Publishing, 2019.

3 World Bank. Deepening health reform in China: building high value-based service delivery-policy summary (English), 2016. Available: http://documents.worldbank.org/curated/en/ 800911469159433307/Deepening-health-reform-in-China-buildinghigh-quality-and-value-based-service-delivery-policy-summary [Accessed 10 Oct 2020].

4 Zhai T, Goss J, Li J. Main drivers of health expenditure growth in China: a decomposition analysis. BMC Health Serv Res 2017;17:185.

5 Moore FD. Small area variations studies: illuminating or misleading? Health Aff 1985:4:96-101.

6 Goodman DC. Unwarranted variation in pediatric medical care. Pediatr Clin North Am 2009;56:745-55.

7 Hendrik J. Health insurance status and physician-induced demand for medical services in Germany: new evidence from combined district and individual level data. DIW Berlin: German Institute for Economic Research, 2007.

8 Wennberg JE. Practice variations and health care reform: connecting the dots. Health Aff 2004;Variation:VAR-140-VAR-144.

9 Silverman EM, Skinner JS, Fisher ES. The association between forprofit hospital ownership and increased Medicare spending. N Engl J Med 1999;341:420-6.

10 Goodman DC, Mick SS, Bott D, et al. Primary care service areas: a new tool for the evaluation of primary care services. Health Serv Res 2003;38:287-309.

11 Klauss G, Staub L, Widmer M, et al. Hospital service areas - a new tool for health care planning in Switzerland. BMC Health Serv Res 2005;5:33.

12 Committee on Geographic Variation in Health Care Spending and Promotion of High-Value Care; Board on Health Care Services; Institute of Medicine. Variation in health care spending: target decision making, not geography. Washington (DC: National Academies Press (US), 2013. https://www.ncbi.nlm.nih.gov/books/ NBK201647/

13 Jia P, Wang F, Xierali IM. Evaluating the effectiveness of the hospital referral region (HRR) boundaries: a pilot study in Florida. Ann GIS 2020;26:251-60.

14 Welch WP, Miller ME, Welch HG, et al. Geographic variation in expenditures for physicians' services in the United States. N Engl J Med 1993:328:621-7.

15 Wennberg JE GJ, Gittelsohn. Small area variations in health care delivery. Science 1973;182:1102-8.

16 Wennberg JE, Fisher ES, Skinner JS, et al. Extending the P4P agenda, part 2: how Medicare can reduce waste and improve the care of the chronically ill. Health Aff 2007;26:1575-85.

17 Zhang Y, Baik SH, Fendrick AM, et al. Comparing local and regional variation in health care spending. N Engl J Med 2012;367:1724-31.

18 Wennberg JE, Fisher ES, Sandra SM. The care of patients with severe chronic illness: an online report on the Medicare program by the Dartmouth atlas project, 2006. Available: http:// wwwdartmouthatlasorg/atlases/2006_Chronic_Care_Atlaspdf [Accessed 12 Oct 2000].

19 Detsky AS. Regional variation in medical care. N Engl J Med 1995;333:589-90.

20 Goody B. Defining rural hospital markets. Health Serv Res 1993;28:183-200.

21 Jia P, Xierali IM, Fahui W. Evaluating and re-demarcating the hospital service areas in Florida. App/ Geogr 2015;60:248-53.

22 Makuc DM, Haglund B, Ingram DD, et al. The use of health service areas for measuring provider availability. $J$ Rural Health 1991;7:347-56

23 Yip W, Fu H, Chen AT, et al. 10 years of health-care reform in China: progress and gaps in universal health coverage. Lancet 2019;394:1192-204.

24 Jia P, Wang F, Xierali IM. Using a Huff-based model to delineate hospital service areas. The Professional Geographer 2017;69:522-30.

25 Department of Urban Surveys of national bureau of statistics of China. China City and social and economic statistical yearbook 2019. Beijing: China Statistics Press, 2019.

26 Wang X, Yang H, Duan Z, et al. Spatial accessibility of primary health care in China: a case study in Sichuan Province. Soc Sci Med 2018;209:14-24.

27 Goodman DC, Wennberg JE. Maps and health: the challenges of interpretation. J Public Health Manag Pract 1999;5:xiii-xvii.

28 Guagliardo MF, Jablonski KA, Joseph JG, et al. Do pediatric hospitalizations have a unique geography? BMC Health Serv Res 2004;4:2.

29 Onega T, Tosteson TD, Wang Q, et al. Geographic and sociodemographic disparities in PET use by Medicare beneficiaries with cancer. J Am Coll Radiol 2012:9:635-42. 
30 Ricketts TC, Belsky DW. Medicare costs and surgeon supply in hospital service areas. Ann Surg 2012;255:474-7.

31 Baicker K, Chandra A. Medicare spending, the physician workforce, and beneficiaries' quality of care. Health Aff 2004;Web Exclusives:W4-184-W4-197.

32 Fisher ES, Wennberg DE, Stukel TA, et al. Variations in the longitudinal efficiency of academic medical centers. Health Aff 2004; Variation:VAR-19-VAR-32.

33 Fisher ES, Wennberg DE, Stukel TA, et al. The implications of regional variations in Medicare spending. Part 1: the content, quality, and accessibility of care. Ann Intern Med 2003;138:273-87.

34 Gerdtham UG, Jönsson B. Chapter 1 - International comparisons of health expenditure: Theory, data and econometric analysis. In: Handbook of health economics. Elsevier, 2000: 11-53.

35 Gerdtham UG, Jönsson B, MacFarlan M, et al. The determinants of health expenditure in the OECD countries: a pooled data analysis. Dev Health Econ Public Policy 1998;6:113-34.

36 Rushing WA. The supply of physicians and expenditures for health services with implications for the coming physician surplus. $J$ Health Soc Behav 1985;26:297-311.
37 Fisher ES, Wennberg JE. Health care quality, geographic variations, and the challenge of supply-sensitive care. Perspect Biol Med 2003;46:69-79.

38 National State Council of China. Several options on promoting the development of health services, 2013. Available: http://www.gov.cn/ zhengce/content/2013-10/18/content_6067.htm

39 Connor RA, Feldman RD, Dowd BE. The effects of market concentration and horizontal mergers on hospital costs and prices. Int J Econ Bus 1998;5:159-80.

40 Kim SJ, Park E-C, Yoo K-B, et al. The association of market competition with hospital charges, length of stay, and quality outcomes for patients with joint diseases: a longitudinal study in Korea. Asia Pac J Public Health 2015;27:195-207.

41 Kessler DP, McClellan MB. The effects of hospital ownership on medical productivity. Rand J Econ 2002;33:488-506.

42 Barro JR, Huckman RS, Kessler DP. The effects of cardiac specialty hospitals on the cost and quality of medical care. $J$ Health Econ 2006;25:702-21.

43 Bjorvatn A. Private or public hospital ownership: does it really matter? Soc Sci Med 2018;196:166-74. 